\title{
Analysis of Compression Pad Cavities for the Orion Heatshield
}

\author{
Richard A. Thompson ${ }^{*}$, Victor Lessard ${ }^{\dagger}$, Thomas Jentink ${ }^{\ddagger}$, and E. Vincent Zoby ${ }^{\S}$ \\ NASA Langley Research Center, Hampton, Virginia
}

\begin{abstract}
Current results of a program for analysis of the compression pad cavities on the Orion heatshield are reviewed. The program was supported by experimental tests, engineering modeling, and applied computations with an emphasis on the latter presented in this paper. The computational tools and approach are described along with calculated results for wind tunnel and flight conditions. Correlations of the computed results are shown which can produce a credible prediction of heating augmentation due to cavity disturbances. The models developed for use in preliminary design of the Orion heatshield are presented.
\end{abstract}

\section{Nomenclature}

$\begin{array}{ll}\mathrm{h} & =\text { heat transfer coefficient } \\ H & =\text { cavity depth } \\ L & =\text { cavity length (diameter) } \\ p & =\text { pressure } \\ q & =\text { heat transfer rate } \\ \mathrm{Re} & =\text { Reynolds number } \\ \mathrm{R} & =\text { heatshield radius } \\ \mathrm{S} & =\text { surface distance } \\ t & =\text { time } \\ T & =\text { temperature } \\ \mathrm{x}, \mathrm{y}, \mathrm{z} & =\text { coordinate directions } \\ \alpha & =\text { angle of attack } \\ \delta & =\text { boundary layer thickness } \\ \theta & =\text { momentum thickness }\end{array}$

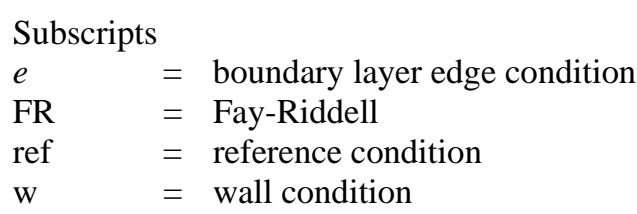

\section{Introduction}

$\mathrm{T}$

he NASA Constellation program [Ref. 1] aims to expand manned exploration of space and, to support this mission, the Orion crew capsule is being designed for return from low Earth orbit and the moon. This capsule-shaped command module (formerly known as the Crew Exploration Vehicle or CEV) shares many traits with the Apollo vehicle from nearly two generations ago. Preliminary design of the Orion module and heatshield has leveraged technology and design solutions from Apollo wherever possible. One example is the support of mechanical loads between the command module (Orion) and service module when stacked for launch. In this configuration, launch loads must transfer through connections on the bottom of the capsule which penetrate though the windward reentry heatshield. Lightweight thermal protection materials for the heatshield do not possess the mechanical strength for support so, like Apollo, the Orion loads are transferred through densified compression

\footnotetext{
* Engineer, Aerothermodynamics Branch, MS 408A, Senior Member AIAA.

${ }^{\dagger}$ Engineer, Gentex Systems, MS 408A, Senior Member AIAA.

${ }^{\ddagger}$ Engineer, AMA, Inc., MS 408A.

${ }^{\S}$ Senior Research Engineer, Aerothermodynamics Branch, MS 408A, AIAA Fellow.
} 
pads embedded within the windward heatshield. A seamless integration of the compression pads within the heatshield would be ideal; however, the need for mechanical connections and even different rates of material ablation dictate some form of surface discontinuity. The presence of these singularities on the heatshield (whether protrusions or recessed cavities) will disturb the smooth flow over the vehicle and result in increased heating both locally and downstream of the pad. The photograph in Figure 1 shows how the Apollo compression pads were recessed into the windward heatshield to form circular cavities. The disturbances caused by these cavities resulted in augmented heating which is discernable in the photograph by the discolored heatshield in areas around the pads. The purposes of the present work are to understand how the pad design affects the flow disturbance and to determine the magnitude and extent of heating augmentation for proper thermal protection system (TPS) sizing on Orion.

Investigation of the aerothermal environments related to the Orion compression pads has been conducted by the CEV Aerosciences Project (CAP) team through a combination of experimental tests, computational simulation, and engineering modeling. An experimental test plan was developed to aid in concept evaluation and to provide validation data for computational fluid dynamics (CFD) of both laminar and turbulent flows. An early compression pad concept is presented in Fig. 2 where the pad geometry forms an annular ring on the heatshield surface and the tension tie connection between capsule and service module lies outside the pad. Other configurations evaluated by CAP included cylindrical cavity recesses and pad protrusions which could occur if the surrounding heatshield ablated faster than the pad.

The configuration shown in Fig. 2, along with other pad shapes and tension tie placements were screened in an experimental survey by Liechty [Ref. 2]. Experimental results for cylindrical pad geometries were obtained by Hollis [Refs. 3,4] where both recessed and protruding pads of varying height and depth were tested. CFD calculations with protruding pads were also performed to characterize the flow disturbance and augmented heating at flight conditions. Both experiment and prediction indicated that conditions where the pads protruded were not desirable since they caused high heating augmentation (up to five times the normal level) on the heatshield downstream of the pads. After exploration of alternate concepts, the Orion design was narrowed to follow an approach similar to Apollo by recessing full circular compression pads into the surface.

Figure 3 illustrates the current layout of compression pads on Orion. There are six pads spaced symmetrically around the center axis at a radial distance of approximately $81 \%$. The Orion design utilizes a carbon phenolic pad material (like Apollo), however, both the Apollo-era Avcoat and a newer phenolic impregnated carbon ablator (PICA) material [Ref. 5] have been considered as the primary TPS material for the

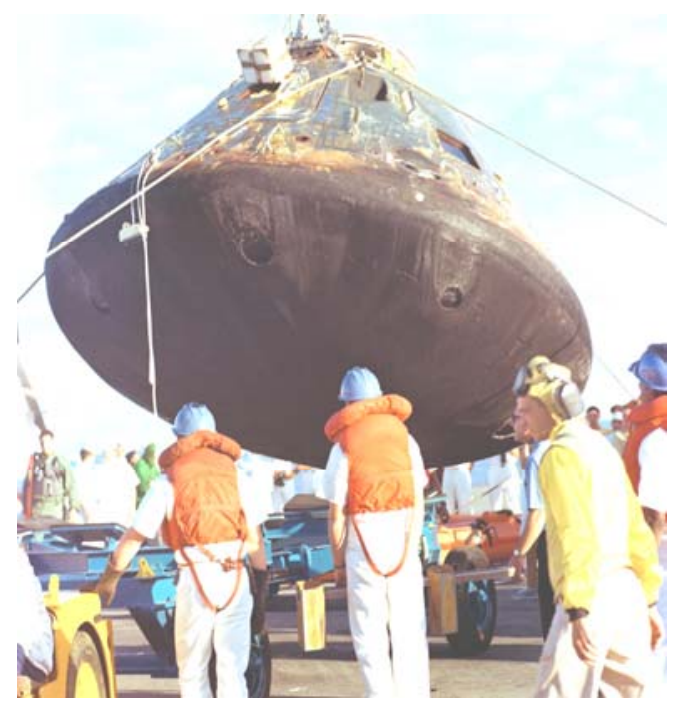

Figure 1. Apollo compression pads after reentry

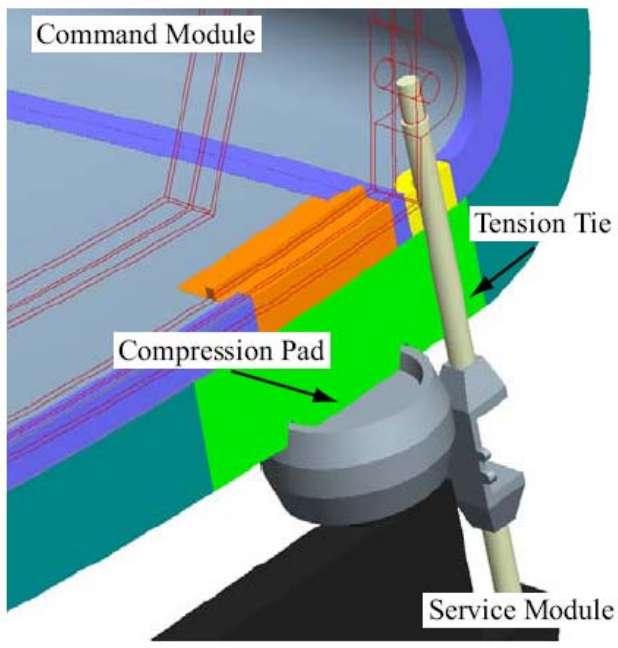

Figure 2. A conceptual design for Orion compression pad

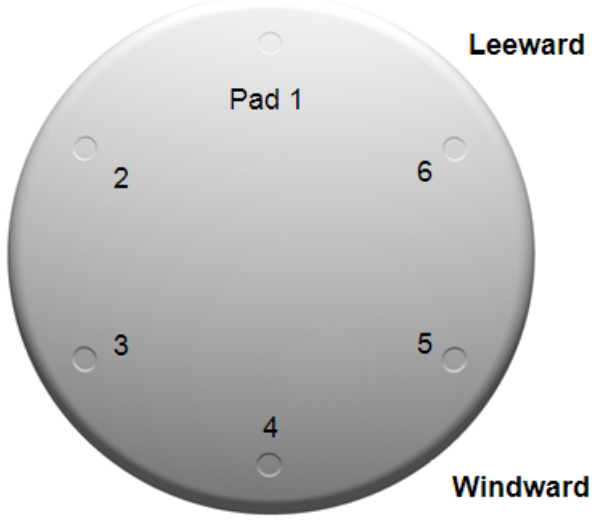

Figure 3. Preliminary layout of Orion compression pads 
heatshield. PICA is more fragile and has significantly higher recession rates than Avcoat and carbon-phenolic. It follows that the PICA design results in a thicker heatshield layer than Avcoat and, as a consequence, the initial cavity depths are larger to prevent the pads from protruding as the surrounding TPS ablates during reentry. The increased cavity depths for a PICA heatshield introduced the likelihood of increased heating augmentation and raised operational concerns for mating and undocking the command and service modules as well. Beveling the cavity sidewalls provided extra clearance without increasing the compression pad diameter (and increasing weight) and so that feature has been incorporated in the baseline design. It was also recognized that the beveled sidewalls might reduce flow disturbances due to the cavities.

A cross sectional view of a typical Orion compression pad cavity is presented in Fig. 4 which shows both the actual geometry and a simplified model that has been used in wind tunnel tests and CFD analyses to date. In the simplified approach, the beveled sidewalls are modeled but the cavity floor is treated as smooth without the presence of tension ties or locking groove. These missing features will be investigated as part of an upcoming experimental test but they have been assumed to be second order effects in the current work. It should be noted that the variable cavity depth depicted in Fig. 4 is the result of a variable TPS thickness and has been modeled in CFD calculations.

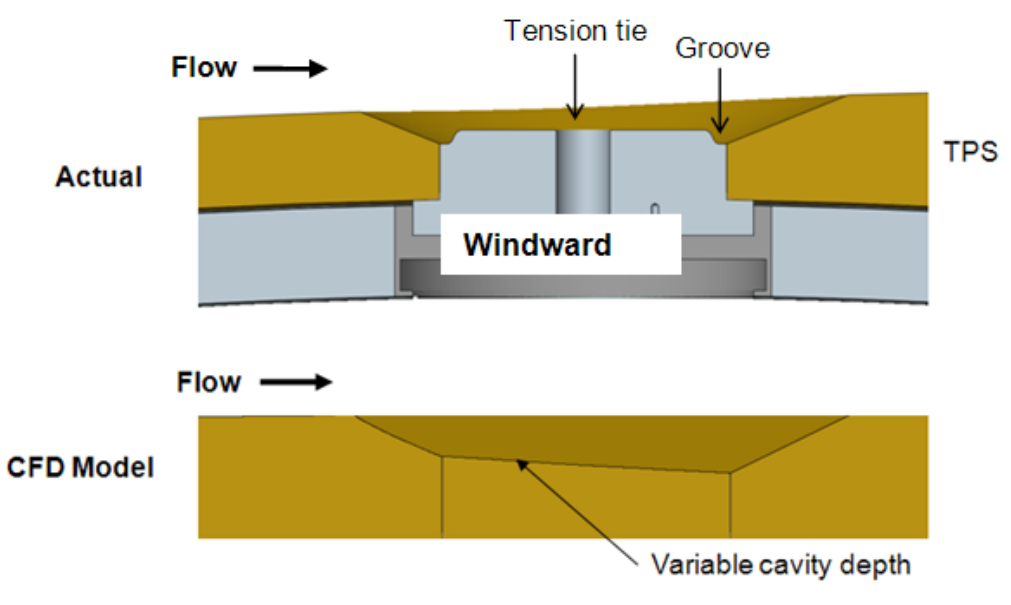

Figure 4. Cross-section of Orion compression pad

From an aerothermodynamic point of view, the environment associated with the Orion compression pads is primarily characterized by cavity flow in the subsonic or low supersonic flow field of the blunt heatshield shape. Numerous investigations of cavity flow have been undertaken in the past and a classification of the flow physics has evolved which is primarily dependent on the ratio of cavity length to depth $(\mathrm{L} / \mathrm{H})$. In general, for a "closed" cavity with $\mathrm{L} / \mathrm{H}>13$ the incoming flow is able to turn into the cavity and attach to the floor before exiting at the downstream end [Ref. 6]. In this case, the downstream wall acts as a forward facing step and the whole flowfield is generally steady though regions of separated flow can exist at both the incoming and downstream ends. For an "open" cavity with smaller values of $\mathrm{L} / \mathrm{H}$ (less than 10), the length is sufficiently short or depth is sufficiently large that the flow does not attach to the floor though it will impinge on the downstream lip. The flow in this case may be unsteady. A zone of transitional cavity flow exists at intermediate values $(10<\mathrm{L} / \mathrm{H}<14)$ and the flowfield may oscillate between the open and closed state. In most cases, the computed flow in the compression pad cavities on Orion at flight conditions has been predicted to be "closed" and steady in nature although a few conditions resulted in oscillating (non-converged) solutions.

The state of the boundary layer (laminar, turbulent, or transitional) may both affect and be influenced by the type of cavity flow (open or closed). An assumption for Orion design is that the flow will be turbulent over the heatshield due to the presence of ablation and the roughened surface that results. Both fully laminar and fully turbulent flows have been considered in the computations performed as part of this work. Additional experimental data is forthcoming which should strengthen the validity of the turbulent results and should provide information about the case when transition occurs inside the cavity. One area that has not been modeled or tested in this work is the effect of ablation of the heatshield and compression pad on the cavity flow and heating augmentation. It is assumed that the effect is second order and would not adversely alter the heating augmentation.

This paper gives an overview of the work completed to analyze the effects of compression pad cavities on the Orion heatshield and presents the results in a form that was useful for TPS design. The paper has the nature of a status report since additional maturation is planned prior to a preliminary design review. In particular, there is limited validation of CFD results presented in this paper but additional experimental tests are underway and planned which will address the validation concerns. The following sections outline the computational approach and present results obtained from application CFD to model the compression pad cavities. Results from validation studies and parametric CFD analyses are shown and correlations of results leading to development of an engineering level 
model are discussed. Finally, a set of functional relationships based on the CFD results that can be used to predict the augmented turbulent heating induced by cavities in the Orion heatshield are described.

\section{Analysis Tools}

\section{A. Computer Code}

The LAURA (Langley Aerothermodynamic Upwind Relaxation Algorithm) code [Ref. 7] uses a finite-volume shock-capturing approach to solve steady viscous and inviscid flow problems. The algorithm incorporates pointimplicit or line implicit relaxation schemes to obtain solutions efficiently on multi-processor and massively parallel computers. The code has been successfully applied to a wide range of hypersonic vehicles and flight conditions during its evolution over the past 15 years. LAURA includes models for perfect gas, equilibrium air, and thermal and chemical nonequilibrium air in addition to models for $\mathrm{CF}_{4}$ and a Mars atmosphere. An important feature of the code is the ability to perform one-dimensional grid adaption in parallel with the solution to resolve high gradients in the boundary layer and across a bow shock. For the applications in this work, the full Navier-Stokes equations were solved and an eigenvalue limiter of 0.3 was used in the code. Turbulent calculations were done using the CebeciSmith algebraic turbulence model. The computational grids in every case were adapted such that the cell Reynolds number at the wall was $\mathrm{O}(1)$ and solution (iterative) convergence was obtained when changes in surface heating were less than $0.1 \%$ after 5000 iterations. All calculations at flight conditions assumed a 5-species air model in chemical nonequilibrium but thermal equilibrium. The surface was assumed to be "super" catalytic to dissociated species so that species mass fractions returned to freestream values. The wall temperatures in each case were obtained using an equilibrium radiative wall condition

\section{B. Grid Generation}

As mentioned in the introduction, protruding pads, cylindrical cavity geometries (90-deg sidewalls), and cavities with beveled side walls have all been studied as part of this work. Different grid systems were developed to model the different geometries to obtain CFD solutions. A multi-block O-type grid topology was used for protuberances and cavities with 90-deg sidewalls as shown in Fig. 5 where the topology of the blocks inside the cavity or surrounding the protrusion matches the encompassing grid. The figure illustrates a grid that was constructed to match a 3.54\% wind tunnel model that had three recessed pad cavities and three protruding pads. In the experimental tests Refs. [3,4] the model was rotated to place the cavities and protrusions at different locations and several pad heights and cavity depths were tested. Only a subset of those parameters was modeled for CFD. In the final grid, there were 452 blocks with a total of 1.2 million points and all block dimensions were equal for load balancing on a parallel computer.

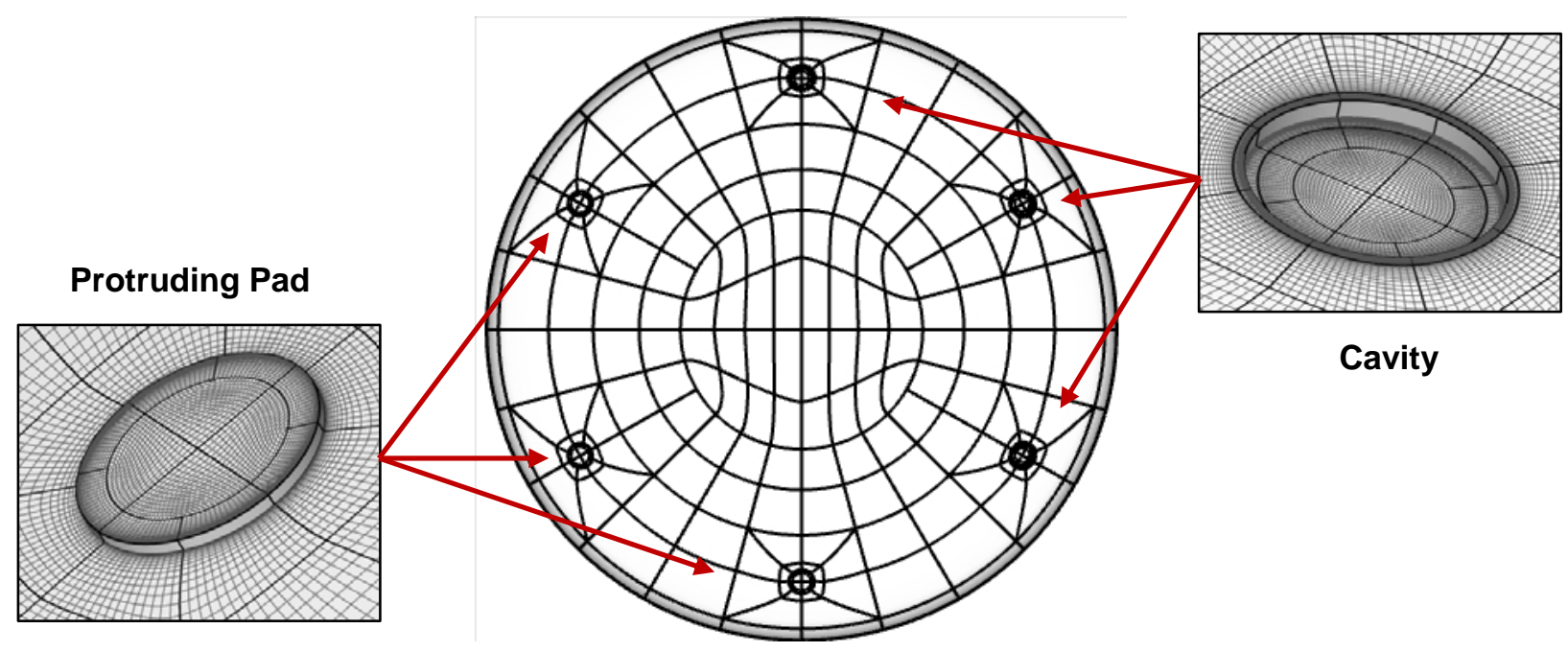

Figure 5. Grid topology and meshes for protrusions and cavities with 90-deg sidewalls 
A new method for generating the CEV heatshield grid with recessed compression pads was developed to expedite grid generation for the cases with beveled sidewalls. A requirement of this method was that the grid topologies for the smooth heatshield and heatshield with compression pad cavities were identical. In either case, the location of each compression pad was isolated within an O-type topology on the surface similar to that shown in Fig. 5. A beveled cavity grid was then created from the smooth grid by first selecting two grid lines ("rings") in the O-topology to define perimeters for the cavity floor and bevel edge. The cavity floor was then depressed into the surface to form the bevel between the two selected "rings" as shown in Fig. 6. The volume grid was reattached to the surface with the condition of orthogonal boundary cells enforced through elliptic smoothing. Finally, the grid was redistributed based on the original or adapted grid distribution. The family of concentric grid lines in the O-topology was constructed a priori such that a range of bevel angles and cavity depths could be

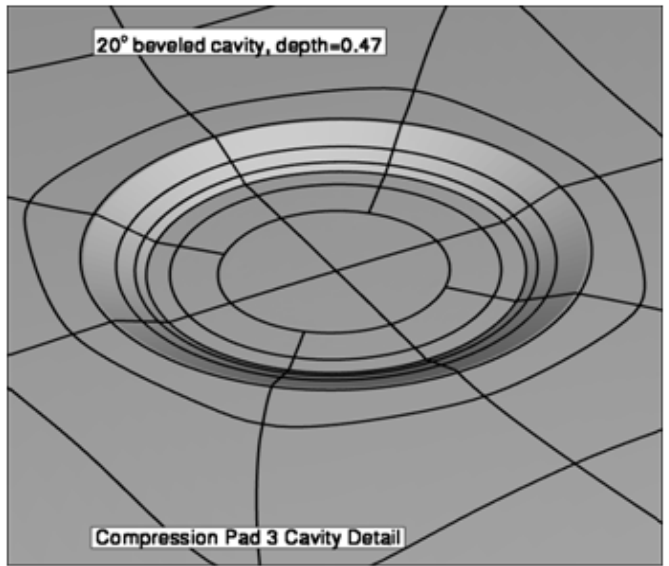

Figure 6. CFD grid topology for modeling beveled compression pad cavities modeled from the same baseline grid with the freedom to have different settings at each pad location. This same approach was implemented for bevel cavities with both constant and variable depths across the cavity floor. The difference in the variable depth modeling is that the grid line "rings" were oval shaped instead of circular and the bevel angle varied \pm 1.5 deg around the cavity as occurs on the actual geometry. When automated, the method proved efficient with turn-around time for grid generation reduced from 4-5 days to less than 10 minutes. In the final grid system, a total of 280 blocks were used to model one-half of the CEV forebody with four compression pads (symmetry assumed). The grids contained 7.8 million points which were equally distributed between blocks for load balancing.

\section{Postprocessing}

The surface heating augmentation factor (AF) is computed as the ratio of heating in the presence of a recessed compression pad cavity to the heating on a smooth OML surface. Laminar and turbulent AF values presented in this paper are computed relative to the laminar and turbulent smooth OML calculations, respectively. In an approach following Ref. 4, the areas of augmented heating in and around the compression pad cavity were divided into three zones - cavity floor, bevel sidewall, and surrounding OML. Within each zone, an area weighted average and maximum value of heating augmentation factor was obtained from CFD. Average values for the bevel and OML zones were computed to give some measure of the heating "footprint" caused by the cavity disturbance. This footprint was determined as the area where the AF values were within $70 \%$ of mean deviation from the maximum within the zone. This method, although somewhat subjective, allowed for a consistent way to compute a conservative average value for the relevant heating load on the thermal protection system. The cavity floor average was computed over the entire floor because the "footprint" area was typically small and essentially captured by the maximum value. Figure 7 illustrates the three zones and area averaging and for a typical case.

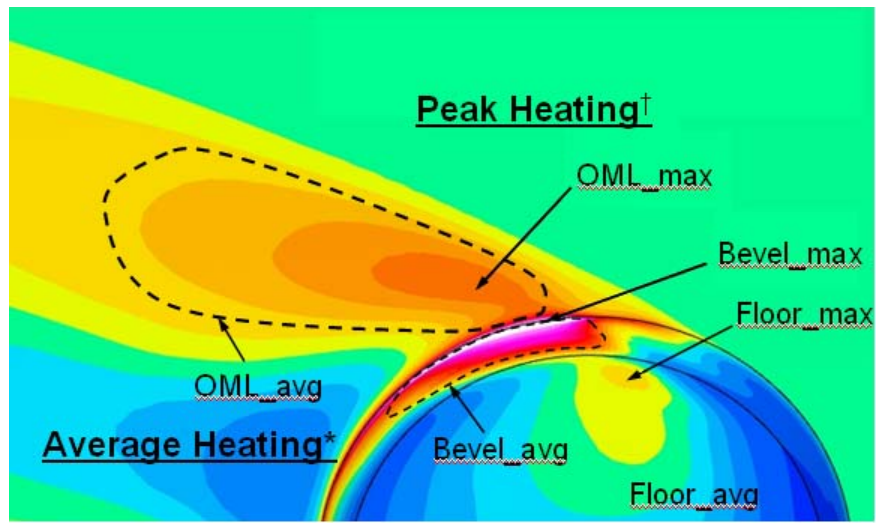

Figure 7. Maximum and average augmentation factors (AF) for three zones 


\section{Results}

\section{A. Recessed Cavities}

While the Orion compression pad design has evolved to a beveled sidewall for the pad cavities, the initial test data was obtained with cavities formed with 90-deg sides. These early tests provided data for studying the effects of recessed and protruding pads on the surface heating including variations in cavity depth and diameter. The data provided guidance on pad placement and has supplied the CAP team with validation data for CFD comparison. Figure 8 shows a comparison of this data with a CFD prediction for the case of compression pad cavities. The experiemental measurements were obtained using phosphor thermography to provide a global map of the heat transfer which is normalized by a reference stagnation value. For comparison, the CFD contours were normalized by the same value and plotted with an identical color map and matching view angle. The experimental image in Fig. 8(a) was taken from a position aligned with the model centerline and looking at a downward angle toward the Pad 1 location. A fair view of the surface heating at Pads 2 and 6 is also shown while the image (data) quality at pad locations 3 and 5 is deteriorated. There is a large difference in heating observed at the Pad 2 and 5 locations because the experimental model was rotated differently than the CFD so a cavity was tested where a protrusion was modeled and vice-versa. Other than these locations, there appears to be good qualitative agreement in heating between experiment and prediction, especially for the Pad 1 location. The level of agreement is shown more clearly in Fig. 8(b) where a zoomed view of the area around Pad 1 is shown. The CFD prediction reveals a narrow region of high heating along the cavity lip which is not evident in the test image; however, the magnitude and extent of augmented heating on the surface downstream of the pad are very similar.

A qualitative comparison of the predicted and measured heating for this location is presented in Fig. 9. In this figure, the heat transfer distribution is plotted along the centerline passing through Pad 1 and extending downstream to the heatshield shoulder region. The data measured at five Reynolds number conditions is plotted along with the predicted levels for the lowest and highest Re condition. The experimental data and predictions for all Reynolds numbers collapse along a single curve in the region upstream of the cavity $(\mathrm{z} / \mathrm{R}<0.75)$ as expected for laminar flow. The data and predictions also nearly collapse inside the cavity with the exception of measurements at the highest two Reynolds numbers. Downstream of the cavity ( $\mathrm{z} / \mathrm{R}>0.85$ ), the measured data shows a clear trend of increasing heating augmentation with increasing Reynolds number. The two CFD predictions also exhibit this trend with the prediction at the lowest Reynolds number in excellent agreement with the test data. In contrast, the CFD calculation at the highest Re $\left(1.7 \times 10^{6} / \mathrm{m}\right)$ underpredicts the measured heating by $30-40 \%$. Given the excellent agreement between measurement and prediction at the lower Reynolds number it was concluded that the flow transitioned to a non-laminar state at the higher Re conditions and caused the discrepancy. Turbulent calculations using an algebraic

eddy-viscosity model for the highest Re number case were unsuccessful assuming both fully turbulent flow and flow with transition in the cavity. Those turbulent calculations will be revisited using a credible higher order turbulence model for further validation. 


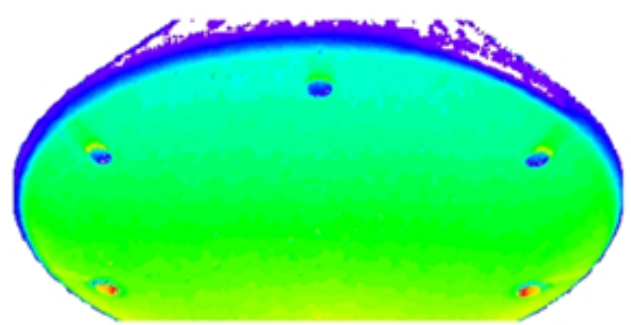

Experiment

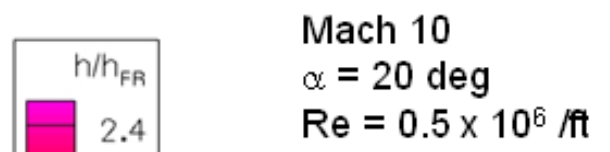

LAURA - fully lamınar

Pad 1

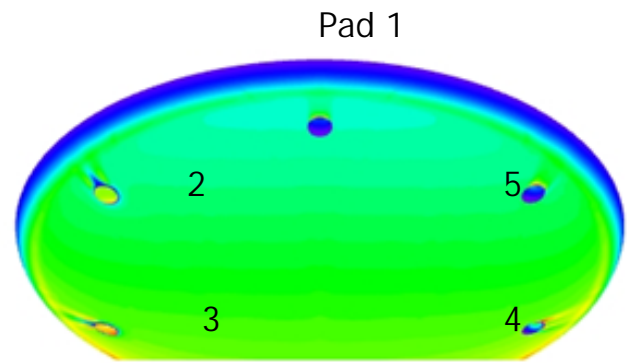

CFD

a) Global image

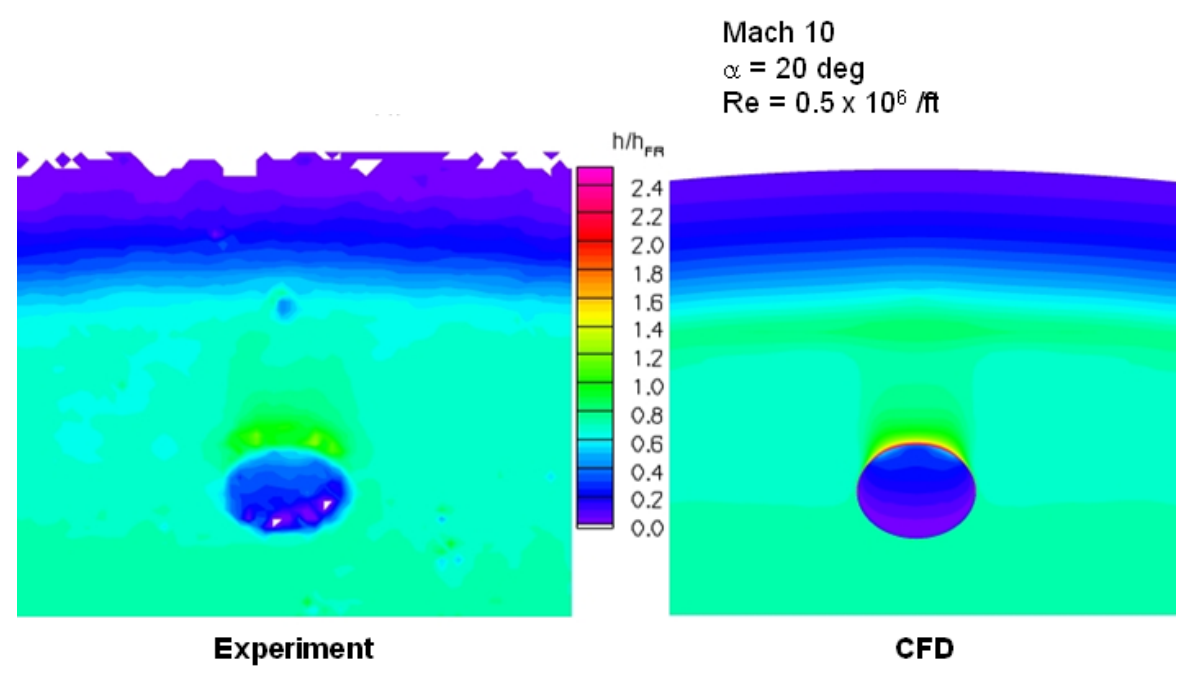

b) Detail at Pad 1 location

Figure 8. Comparison of predicted and measured surface heating 


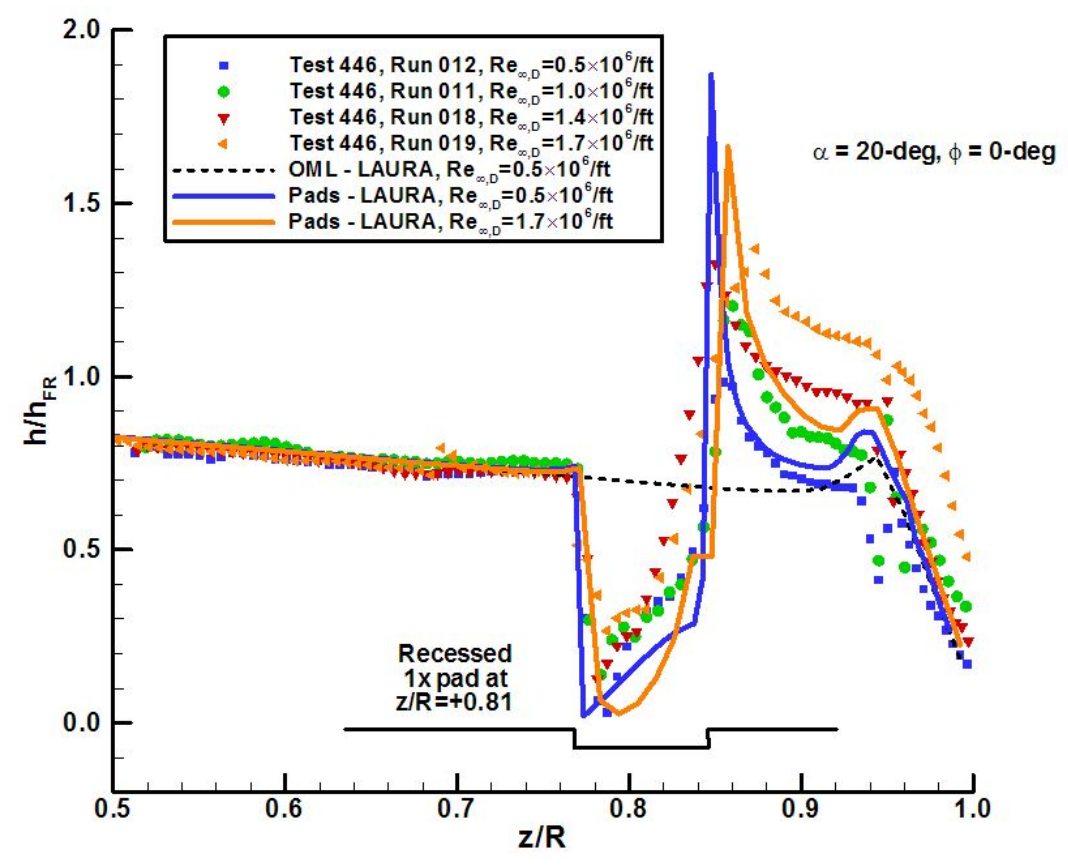

Figure 9. Measured and predicted centerline heating at Pad 1

As mentioned previously, the Orion design has incorporated beveled sidewalls in the compression pad cavities to alleviate docking interference. The beveled walls also afforded an opportunity to minimize the heating disturbance caused by the cavities and so a computational study was performed to investigate the bevel effects on heating. In the study, a range of bevel angles (20,45, and 90-deg) and several cavity depths and angles of attack were considered. The calculations were performed at freestream conditions representative of peak heating in flight and results at the Pad 3 location were used to judge the effects. Previous calculations had shown Pad 3 as the site of the largest heating augmentation and limiting the calculations to that location made the study tractable.

The results indicated that angle of attack variation less than 5 deg had a small effect on the augmented heating while a decrease in cavity depth served to decrease the augmented heating levels and extent of the disturbed flow as would be expected for a shallower cavity. A large effect on heating was computed by changing the bevel angles as shown by the comparison of surface heating contours for the three bevel angles in Fig. 10. A general trend observed is that the maximum heating augmentation is reduced and the area of peak heating moves off the surrounding heatshield onto the beveled sidewall. The area of disturbed flow (and higher heating) downstream of the cavity was made smaller by decreasing the bevel angle. In addition, the heating to the cavity floor was increased by the decreasing bevel angle. In general, the cavity flow is less separated and the external flow is more able to enter the cavity as the sidewall angles are decreased. From a design view, the increased heating on the cavity floor is desirable since the carbon-phenolic pads are better able to withstand the heating than the acreage TPS. It is obvious that a minimum bevel angle would yield the optimum design and this is confirmed in Fig. 11 where the predicted augmentation factors are plotted against the sidewall angle. The figure shows the relationship between augmented heating factor and bevel angle to linearly approach one as the bevel angle approaches zero. Physical constraints of the heatshield limit the minimum angle so the preliminary Orion design incorporates a 20-deg angle bevel in the baseline configuration following these results. 


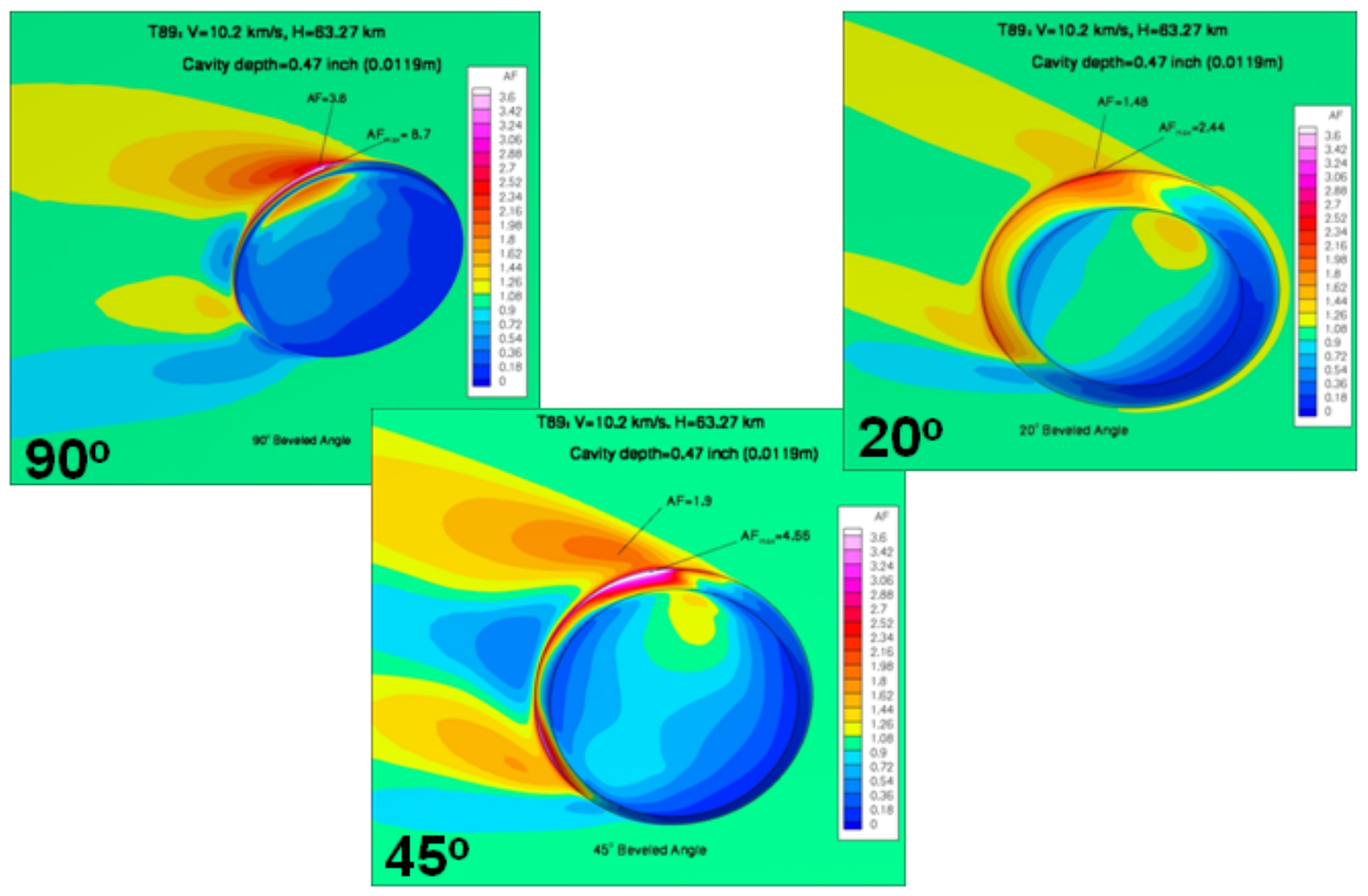

Figure 10. Comparison of surface heating contours for different bevel angles

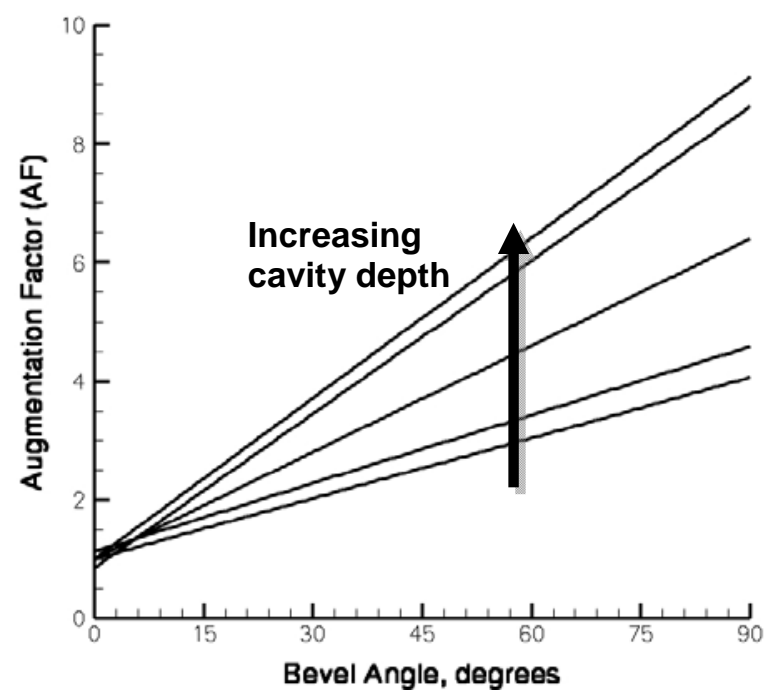

Figure 11. Augmentation factor on sidewall with change in bevel angle

An effort was made to verify grid convergence of the CFD solutions that were used for the parametric study of bevel angle and for the flight cases discussed in the following sections by doubling the grid size. Iterative convergence was assured by following standard practices (see section II.A). In the grid resolution study, the volume cell spacing in all three directions was halved and additional solutions were obtained for two cases where a cavity at the Pad 3 location was modeled. In one case, the grid was doubled throughout the entire domain while the second 
case only doubled the grid in the blocks surrounding and inside the cavity. In comparing the solutions with the refined grids to those obtained with a normal grid density it was observed that the disturbance "footprints" were more sharply resolved as expected but quantitative differences in regions of maximum heating were small. Figure 12 illustrates that point by plotting the ratio of predicted heating obtained using the refined grid to the heating predicted with normal grid spacing. The figure shows the largest differences to occur in the separated flow on the upstream cavity sidewall and floor but the heating on the downstream bevel and cavity lip do not change by more than 5\%. Differences seen in the downstream footprint on Fig. 12 indicate movement of the disturbance by refining the grid but the maximum heating (and augmentation) remained in good agreement.

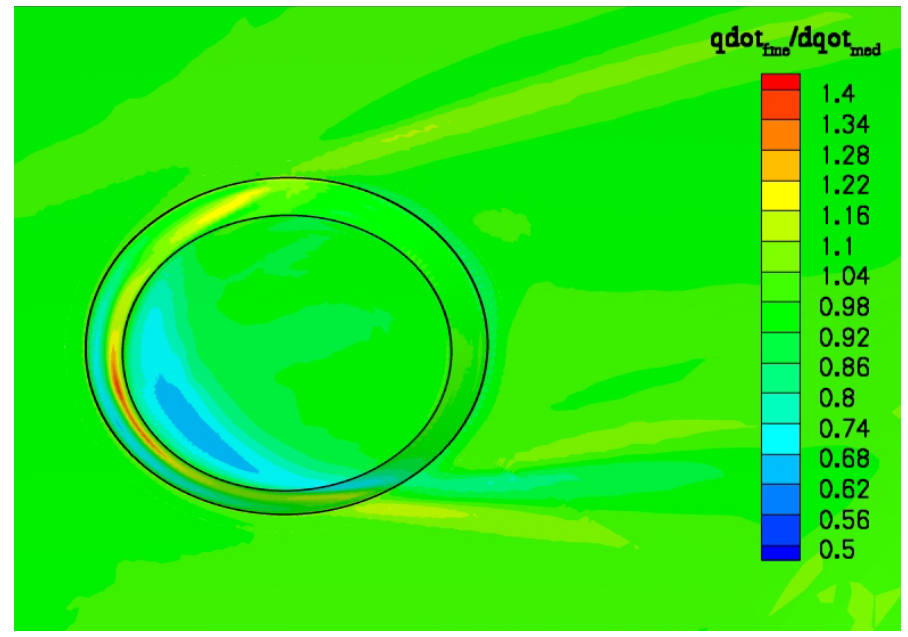

Figure 12. Effect of grid resolution on surface heating

\section{B. Data Correlations}

A number of calculations for recessed pads with various cavity depths were computed subsequent to the parametric study of bevel angles to support the TPS design efforts. An increasing uncertainty in the recession modeling being used for design and the decision to keep two baseline TPS materials (PICA and Avcoat) contributed to the need for more CFD solutions. Refined weights and trajectories also played a role in changing the environments and conditions where it was desired to know the impact on heating augmentation. Given the growing number of computed results it was of interest to understand the trends and correlations of these data for possible extension to other cases where freestream conditions were different, cavity depth or diameter changed, or pad location was altered.

A first attempt at correlating the present results follows the approach developed in Refs. 8 and 9. In that work, tests were conducted on a large number of rectangular cavities on flat plates in support of the Shuttle return-to-flight activities. The conditions for those tests produced flow entering the cavities with supersonic Mach numbers at the boundary layer edge. Data for both laminar and turbulent flow entering the cavity were obtained as well as for flow which transitioned in the cavity. The correlations were found to work well when applied to test data for cavity shapes not used as part of the correlation process. In that work, the augmentation factor for the heating at the cavity endwall was cast in the following functional form:

$$
\begin{aligned}
& X=\ln \left[M_{e}{ }^{\sigma} \operatorname{Re}_{\theta}^{\tau}\left(\frac{L}{\delta}\right)\right] \\
& Y=\ln \left[\left(\frac{H}{\delta}\right)^{\alpha}\left(1+\frac{L}{H}\right)^{\beta} B F\right]
\end{aligned}
$$

A linear curve fit of data plotted in terms of these parameters yields a method to predict the heating augmentation given the local flow conditions and cavity geometry. It was anticipated that the correlation may work for heating 
augmentation on the downstream bevel of the compression pad cavities so the present CFD results were cast in terms of those parameters. The correlation coefficients derived in Ref. 8 were applied to the current laminar data and new coefficients were also derived. Figure 13 shows the laminar correlation using the newly derived coefficients when applied to the whole CFD dataset. It should be noted that all four pad locations are included in this dataset although the same process could be followed for each pad individually. There is a good linear correlation with this dataset $\left(r^{2}=0.97\right)$ and the average error from this function was found to be $15 \%$ when compared to the individual data values. Although not shown here, the coefficients that correlated the turbulent data reported in Ref. 9 were also applied to the present turbulent data but a poor fit was obtained when the whole dataset was included. Improved fits were obtained by separating the data for each pad individually.

A different correlation of the computed data is presented in Fig. 14(a) for laminar flow and Fig. 14(b) for the turbulent results. In both cases the augmentation factor is plotted as a function of $\mathrm{Re}_{\theta}$, $\mathrm{M}_{\mathrm{e}}$, and the ratio of cavity diameter (length) to cavity depth with different exponents being used on the terms depending on the laminar or turbulent state. These individual terms are typical parameters describing cavity flows and were found to correlate the present results well when cast in the form shown. As in the previous approach, linear fits to the laminar and turbulent data were obtained but with a simpler choice of parameters. The correlation factors $\left(\mathrm{r}^{2}\right)$ in these fits were 0.93 (laminar) and 0.89 (turbulent) and, more importantly, the average errors were reduced relative to the previous approach. Here, the average errors were found to be $8 \%$ and $5 \%$ for the laminar and turbulent fits, respectively.

\section{Flight Calculations}

The correlations presented in the previous section appeared to capture the nature of the compression pad augmentation but there was not sufficient confidence to rely on the data fits to provide design information for Orion. Instead, a direct approach of computing the flow (and heating) at points along the flight trajectory was undertaken for conditions spanning the peak heating portion of the trajectory since both the cavity depths and expected augmentation were at their largest through that range. A representative time history for heating at a point on the Orion heatshield is plotted in Fig. 15 along with the variation of cavity depths that were predicted due to

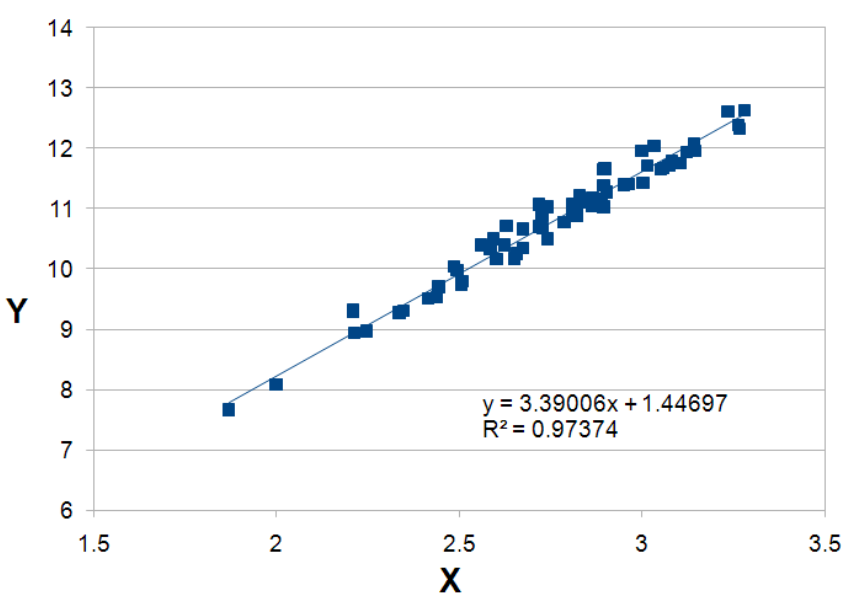

Figure 8. Correlation of present laminar results with approach of Ref. 8

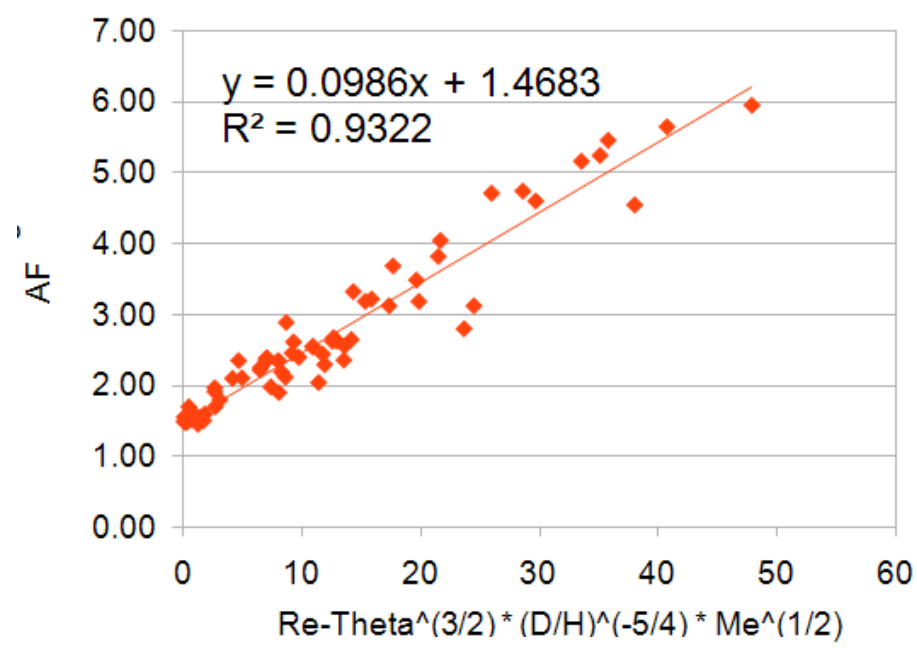

a) Laminar flow

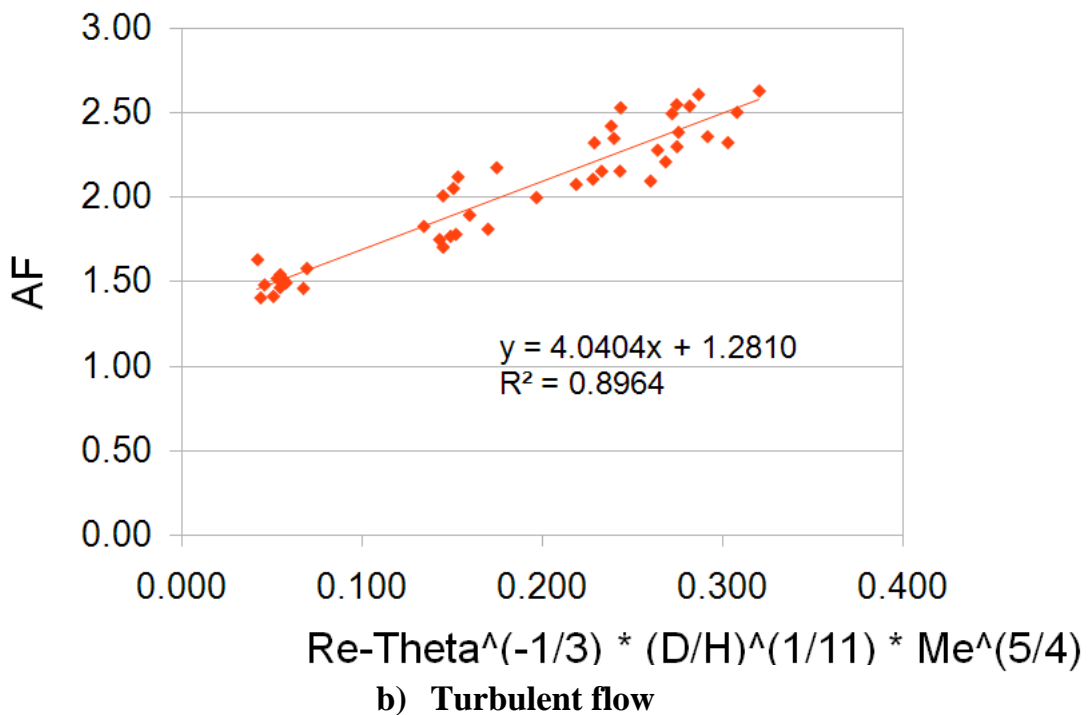

Figure 14. Correlation of compression pad results with present approach 
recession of a PICA heatshield. The curves reflect the double heat pulse associated with a skip reentry and, as designed, the cavity depths approach zero at the second peak. Three trajectory points surrounding the first heat pulse were selected for the CFD solutions as shown on the figure. For a closed cavity flow, the maximum heating augmentation was expected where the ratio of boundary layer thickness to cavity depth $(\delta / H)$ was a minimum. Therefore, the CFD points were subjectively chosen to cover a maximum time period while keeping the ratio to a minimum. It happens that the second point $(\mathrm{t}=85 \mathrm{sec})$ corresponds to peak heating and was also the absolute minimum of the $\delta / \mathrm{H}$ ratio.

The cavity geometry assumed at each trajectory point maintained the shape illustrated in Fig. 4 with the depths changing with time due to ablation of the surrounding surface. Recession of the surrounding heatshield was computed based on heating environments for a smooth OML and did not account for any augmented heating created by the cavity itself. In reality, the surface downstream of the compression pad cavities would experience increased recession due to augmentation so that the relative cavity depths would be shallower than assumed in this approach. Keeping the cavity depths at the maximum levels was believed to be a conservative approach by promoting the largest augmentation.

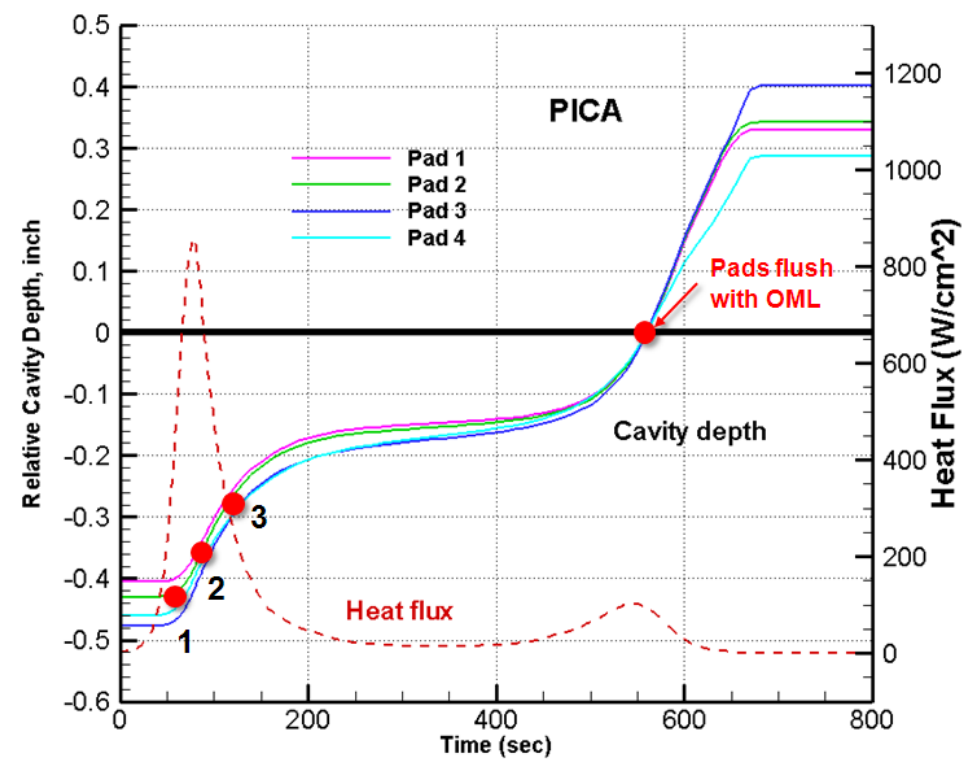

Figure 15. Typical heating profile and cavity depths along reentry trajectory

The CFD solutions calculated at each of the selected trajectory points included cavities with depths for both PICA and Avcoat heatshields for both laminar and fully turbulent conditions. The output from each calculation was processed to find the average and maximum augmentation factors associated with each of the three pad areas depicted in Fig. 7. The processed results were then tabulated versus the trajectory time to yield time-dependent functions of the augmentation factors. A total of 48 relationships were obtained for each of the average and maximum factors for the two TPS materials (PICA, Avcoat), four pad locations (1-4), three pad areas (floor, bevel, OML), and two flow states (laminar, turbulent). In terms of TPS design, the most critical relationship from this work was the augmentation for maximum heating on the cavity bevel sidewall. Figure 16 illustrates the time history of the bevel augmentation factors for both TPS materials. In Fig. 16(a), the augmentation for laminar flow is shown to reach a maximum at the time of peak heating for Pads 1 and 2 but exhibits less variation for Pads 3 and 4 . Cavity depth is also observed to have a significant impact for laminar flow where augmentation factors up to 5.5 are predicted for the PICA heatshield at Pad 1 but only reach 3.5 for Avcoat. In contrast, the results for turbulent flow (Fig. 16(b)) show that the augmentation remained nearly constant over time and almost no difference in augmentation was predicted for the disparate cavity depths associated with PICA and Avcoat. It was also notable that, for the same cavity depths, the turbulent augmentation factors in all of the tabulated relationships were generally equal or less than the laminar values. 
The time dependent augmentation factors obtained is this work were supplied to the Orion program to aid the design and analysis of the TPS and compression pads. In practice, the turbulent factors for maximum bevel heating were used to assess the heatshield and cavity design by performing thermal analyses which showed the carrier structure to remain below temperature limits with the addition of augmented heating.
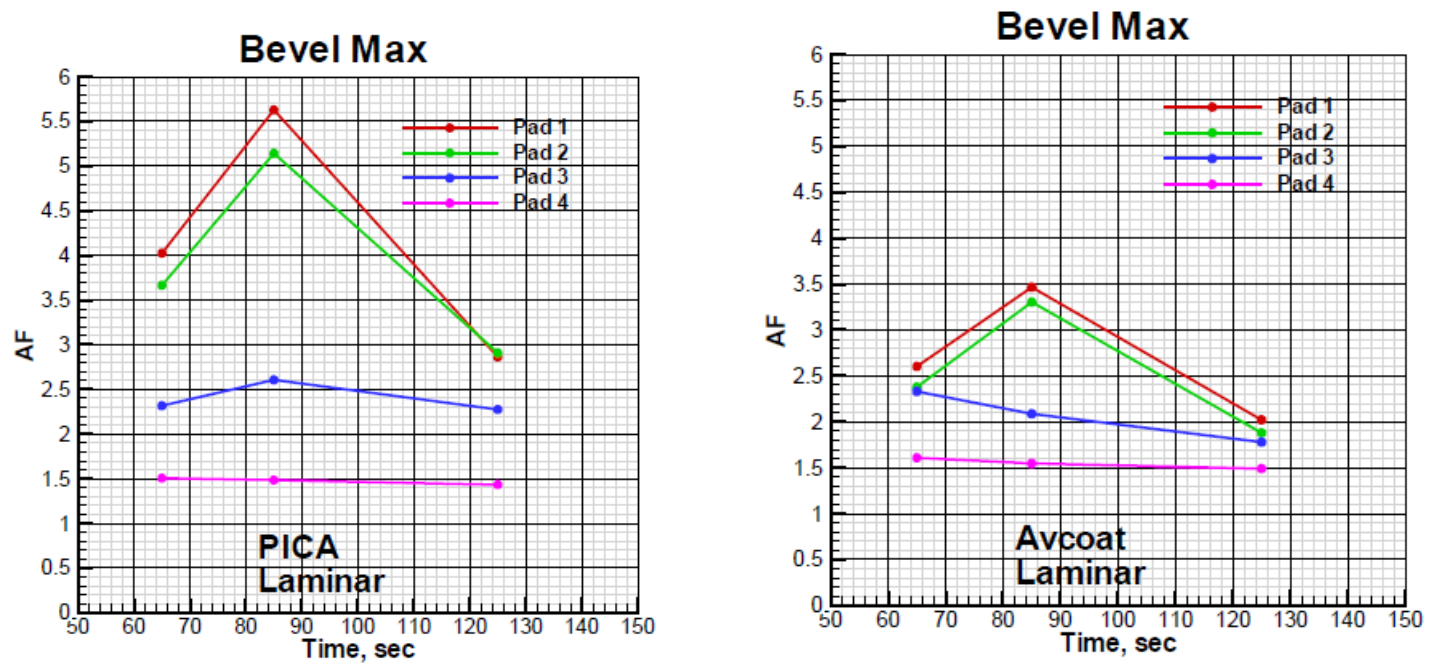

a) Laminar flow
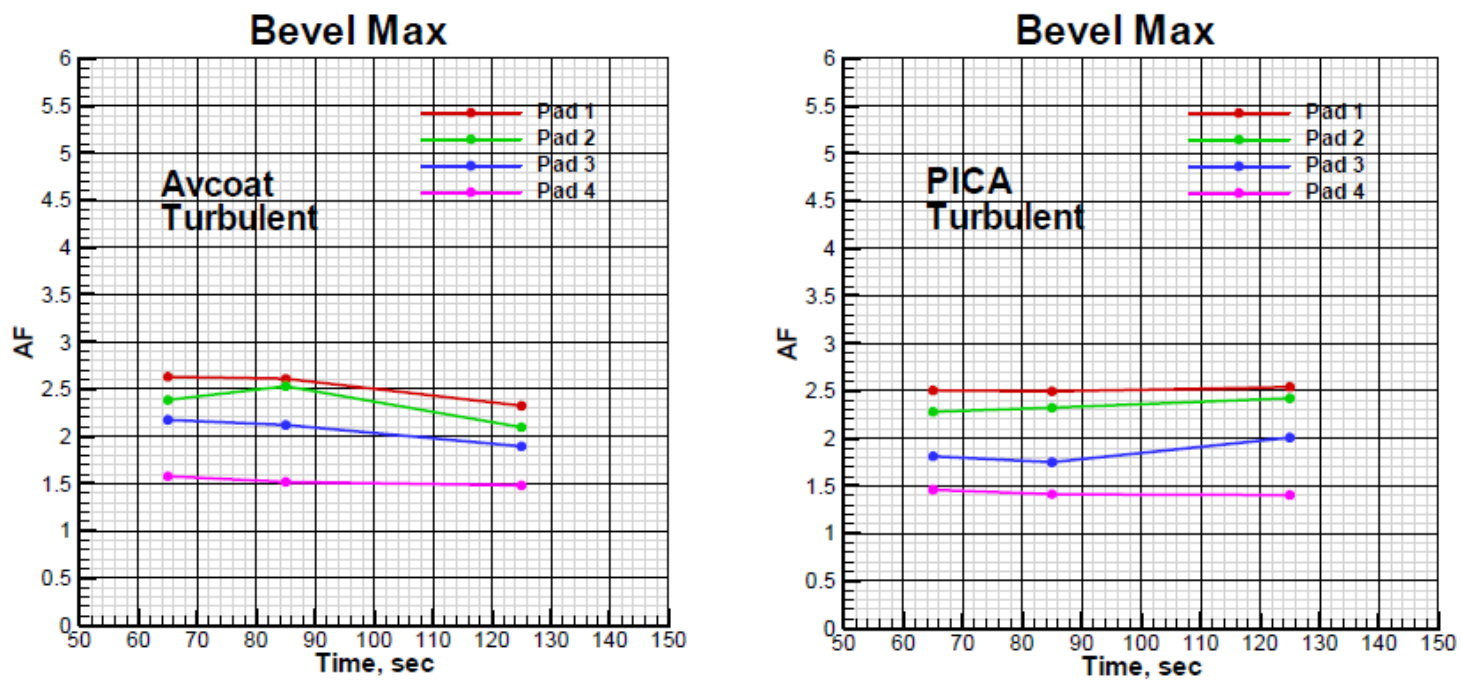

b) Turbulent flow

Figure 16. Predicted time history of heating augmentation

\section{Summary}

A program for analysis of the compression pad cavities on the Orion heatshield was reviewed to show the basis of models used to account for heating augmentation in the design process. To date, the analysis work has supplied parametric information which led to selection of a low 20-deg bevel angle for the cavity sidewalls and has produced the relationships used to predict heating increases from the cavity disturbances. Relations for the maximum heating 
and average heating on the cavity floor, sidewall bevel, and downstream OML were developed for both PICA and Avcoat with both laminar and turbulent flow. Correlations of the heating augmentation were also developed which provide an alternate and credible prediction method. Some validation of the computed works was presented by results of a grid resolution study and by comparison of predictions with experimental data. Additional validation with experimental data will be the focus of future work as tests are completed and more data becomes available.

\section{References}

1. “NASA’s Exploration Systems Architecture Study, Final Report,” NASA TM 2005-214062, November 2005.

2. Liechty, D.S., "Aerothermodynamic Testing of Protuberances and Penetrations on the NASA Cycle I Crew Exploration Vehicle Heat Shield in the NASA Langley 20-Inch Mach 6 Air Tunnel: Test 6918,” NASA EG-CAP-06-155

3. Hollis, B.R., "Aerothermodynamic Testing of Recessed / Protruding Compression Pads on CEV Heat Shield: LaRC 31Inch Mach 10 Air Tunnel Test 446,” NASA EG-CAP-07-165

4. Hollis, B.R., "Aerothermodynamic Testing of Recessed / Protruding Compression Pads on CEV Heat Shield: LaRC 20Inch Mach 6 Air Tunnel Test 6946,” NASA EG-CAP-07-144

5. Tran, Huy K., et. al., “Qualification of the Forebody Heatshield of the Stardust’s Sample Return Capsule,” AIAA Paper 97-XXXX, June 1997.

6. Rossiter, J.E., "Wind Tunnel Experiments on the Flow Over Rectangular Cavities at Subsonic and Transonic Speeds,” RAE Technical Report 64037, 1964.

7. Gnoffo, P. A., “An Upwind-Biased, Point-Implicit Relaxation Algorithm for Viscous, Compressible Perfect-Gas Flows,” NASA TP 2953, 1990.

8. Everhart, J.E., "Supersonic/Hypersonic Laminar Heating Correlations for Rectangular and Impact-Induced Open and Closed Cavities”, AIAA Paper 2008-1283.

9. Everhart, J.E. and Greene, F., “Turbulent Supersonic/Hypersonic Closed- Cavity Heating Correlations,” AIAA Paper 2009-1400, January 2009. 